\title{
La influencia de la experiencia en las competencias investigadoras del profesor universitario
}

\section{The influence of experience on the research competencies of university professors}

\author{
Òscar MAS-TORELLÓ \\ Universidad Autónoma de Barcelona
}

Recibido: Marzo 2014

Evaluado: Junio 2014

Aceptado: Julio 2014

\section{Resumen}

Las instituciones universitarias están en un proceso de transformación, entre otros motivos por la implementación del Espacio Europeo de Educación Superior. Estos cambios provocan alteraciones en el trabajo que desarrolla el profesor universitario, ello conlleva la necesidad de adquirir y/o desarrollar nuevas competencias para atender correctamente las nuevas funciones y tareas profesionales demandadas. Para diseñar planes de formación adecuados a las nuevas necesidades formativas y establecer referentes en los protocolos de acreditación, selección y promoción, es necesario delimitar el nuevo perfil competencial que debe atesorar este profesional para desarrollar convenientemente sus funciones docente, investigadora y gestora, considerando sus diferentes escenarios de actuación profesional y momentos de desarrollo profesional.

Para delimitar el perfil competencial del profesor universitario se ha realizado una investigación fundamentalmente descriptiva que combina metodologías cuantitativas y cualitativas, triangulándose diferentes técnicas (cuestionarios, entrevistas y grupos de discusión) y fuentes de información (profesorado, expertos y alumnos).

Por las lógicas limitaciones de espacio en este artículo únicamente aportamos los datos obtenidos, desde el propio profesor universitario (experto y novel) de las 4 competencias investigadoras delimitadas (diseño, desarrollo y evaluación de proyectos; organización y gestión de eventos científicos; elaboración de material científico; comunicación y difusión de conocimientos científicos) y analizamos los datos más significativos de las 30 unidades competenciales que las componen. Los resultados presentan diferencias significativas en la autopercepción del dominio competencial actual entre profesorado novel y el experimentado. Estos resultados nos ayudan a establecer áreas prioritarias de formación para el profesorado según su etapa de desarrollo profesional.

Palabras clave: competencias, experiencia, universidad, formación, investigación, profesor. 


\begin{abstract}
Implementation of EHEA, among others, motivates a transforming process in universities and promotes changes in the professor's tasks. This events, promote changes to which the university professor has to adapt, meaning this to acquire and to develop new competencies to respond correctly to new professional tasks that are demanded. To design training plans in accordance to the new training needs, and to establish referents in accreditation, selection and promotion protocols appear to be necessary to define the new competences profile professors have to face in order to develop their teaching, research and management tasks suitably, and according to their professional settings and professional development stage.

A qualitative research was developed to define the emerging competence profile. Quantitative and qualitative methods were mixed as well as different tools (questionnaires, interviews and focus groups) and sources (faculty, experts and students).

This article only shows the results obtained from professors (expert and novice) in the 4 research competencies analyzed (design, development and assessment of projects; organization and management of scientific events; development of scientific material; communication and dissemination of scientific knowledges) and the most significant dates of the 30 competency units that compose them.

Results show significant differences of self-perception of current competency domain level between novice faculty and expert faculty. These results help us to establish the priority training areas to the faculty according to their professional development stage.
\end{abstract}

Keywords: competencies, experience, university, training, research, teacher.

Diversos aspectos están modificando el contexto donde el profesor universitario desarrolla su actividad profesional e incrementando la exigencia y control de calidad sobre él (Galán, González y Román, 2012): exigencias de competitividad y productividad concretándose en multiplicidad de evaluaciones/acreditaciones internas y externas, empeoramiento de las condiciones laborales escudándose en la crisis económica, etc. Aunque cabe destacar los cambios propiciados por la convergencia hacia un Espacio Europeo de Educación Superior (EEES), que conllevan nuevos planteamientos en los procesos de enseñanza-aprendizaje (a partir de Tejada, 2005): cambio de paradigma educativo (pasando de centrar la atención en la enseñanzaprofesor a centrarse ahora la atención en el aprendizaje-alumno), cambios estructurales (grados, postgrados, formato en ECTS, etc.) y otros cambios sustantivos (revisión de las titulaciones y materias, revisión de las metodologías y materiales didácticos, diseño y evaluación por competencias, etc.).

Como en cualquier proceso de innovación, cambio y/o reforma universitaria, el profesorado es uno de los elementos nucleares a considerar, no pudiendo desarrollar una concepción de la educación superior y de la investigación de calidad sin incidir de manera clara en este colectivo y en sus competencias.

Las modificaciones del panorama universitario generan, a su vez, la necesidad de delimitar el perfil competencial necesario para que el profesorado desarrolle adecuadamente las nuevas funciones, tareas y roles asignados en este escenario emergente en configuración. Estos acontecimientos nos plantean la necesidad de revisar las implicaciones formativas (inicial y continua) que generan, las 
consideraciones en los protocolos de acreditación/certificación de competencias y en los procesos de selección/promoción de dicho profesorado y, en definitiva, la repercusión en su carrera profesional.

Según los argumentos presentados por el mismo informe del ICE de la Universidad de Zaragoza (2004), podemos afirmar que en este nuevo contexto universitario no bastará con poseer las competencias comunicativas, pedagógicas y curriculares específicas que atesora el profesorado actual, se deberá incidir y adecuar la formación para que este colectivo adquiera unas competencias profesionales básicas más amplias. González Soto (2005), destaca en este contexto la necesidad prioritaria de la formación del personal docente e investigador, pues sin ella, no se producirá cambio alguno, debiendo la universidad centrase en propiciar y potenciar la preparación de su profesorado para la investigación y la docencia.

En el tema que nos ocupa "es importante señalar que no todos los perfiles [para nosotros funciones] del profesorado universitario [docente, investigador y gestor] gozan del mismo reconocimiento ni generan la misma satisfacción laboral... las evaluaciones externas e internas reconocen la producción investigadora como el resultado susceptible de mayor reconocimiento” (Galán, González y Román, 2012, p.135), "la docencia no está bien valorada... el sistema de evaluación del profesorado se basa en la producción científica” por lo que "la investigación da prestigio y es más atractiva” (Tomàs e Ion, 2008, p.140-141) y, como bien indican Tomàs, Castro y Feixas (2012), el doble trato que reciben docencia e investigación y la disparidad en su evaluación genera críticas y tensiones e interferencias entre ellas.

\section{Hacia un perfil docente universitario: funciones, escenarios de actuación y competencias}

Tomàs (2001) afirma que "volver a pensar la Universidad significa reconceptualizar el papel del profesorado, de los estudiantes, de la enseñanza-aprendizaje, de la investigación, del gobierno y la gestión”, significando este replanteamiento de la función docente "dejar el papel de reproductor de conocimiento e ir hacia un orientador de aprendizajes...” ya que, también, se reorienta el aprendizaje de los estudiantes que "debe permitir adquirir conocimientos pero especialmente saberlos buscar, procesar y aplicar” (p.7).

Una segunda función, en la que se centra este texto, es la investigadora donde también los cambios se van sucediendo: fomento de la investigación competitiva, creación de equipos multidisciplinares, integrados a su vez, por miembros de diferentes Universidades, Estados, etc.

Como tercera función que puede desarrollar el profesor universitario no podemos dejar de hacer referencia a la gestión. El personal docente e investigador, como un miembro más de la institución, puede participar activamente en la gestión de su organización, pero debemos alertar que de la lectura de la LRU (1983) y LOU (2001) se desprende que las funciones inherentes al profesor universitario son la docencia y la 
investigación, quedando la gestión como el compromiso personal que cada uno adquiera voluntariamente con la institución donde labora.

Si aceptamos que las competencias profesionales son mucho más que una simple acumulación de contenidos (saber), estando también constituidas por habilidades (saber hacer), actitudes y valores (saber ser y estar), experiencias, aspectos personales, etc., demostrándose su posesión cuando el individuo es capaz de activar todos estos saberes en un contexto laboral específico para resolver óptimamente situaciones y/o problemas propios de su rol, función o perfil laboral; antes de intentar definir parcialmente el perfil competencial del profesor universitario, no podemos eludir establecer sus tres diferenciados (pero íntimamente interconectados e interrelacionados) escenarios de actuación profesional: el contexto general (entorno sociolaboral, profesional, cultural), el contexto institucional (departamento, facultad, universidad) y, por último, el microcontexto aula-seminario-laboratorio.

Es evidente que las funciones del profesor universitario que estamos analizando se entienden, únicamente, si se relacionan con sus escenarios de actuación profesional; "ninguna actividad humana es "context free". Tampoco lo son las acciones profesionales. Todas surgen en un contexto que las condiciona” (Zabalza, 2012, p.22).

Las tres funciones mencionadas cobran una posición relevante según el escenario donde situemos al profesor universitario. Dicho profesor no sólo "actúa" en el microescenario, donde alcanza mayor relevancia la función docente y la investigación sobre su propia docencia y la gestión de los recursos; sino que su actuación se extiende al escenario institucional, donde la gestión y la coordinación son tomados como referentes indiscutibles, pudiéndose considerar de igual modo la investigación y, como no, el contexto sociolaboral y cultural donde de nuevo se prioriza la investigación, relacionada ésta con el desarrollo de convenios, transferencia tecnológica con instituciones externas y, aunque también están presentes la gestión y docencia referentes a las relaciones y actividades extrainstitucionales.

Del mismo modo, la definición del perfil competencial del profesor universitario no puede perder como referente las funciones profesionales que debe asumir (gestión y, especialmente, docencia e investigación), ni los ya mencionados escenarios donde las desarrollará (contexto social, institucional y aula).

Nuestra propuesta de perfil competencial del profesor universitario (Mas, 2009, 2011; Mas y Tejada, 2013; Ruiz, Mas et al., 2008) está conformada por 10 competencias, 6 relacionadas con la función docente (desglosadas en 34 unidaddes competenciales -UC-) y 4 con la función investigadora (desglosadas en 64 UC). Entendiendo, a partir de las aportaciones de diversos autores, una UC como la parte mínima en que se puede fragmentar una cualificación profesional, que sigue teniendo un valor y significado propio en el ámbito laboral, pudiéndose certificar y acreditar su obtención. 


\section{La función investigadora y sus competencias}

El profesor universitario se deberá ocupar y preocupar por investigar, para continuar creando conocimiento científico y mejorar su campo científico, para ofrecer nuevas propuestas metodológicas adaptadas a sus alumnos y materias, para innovar en su realidad y en su contexto, etc. Como indica Iglesia (2011) "cualquier docente debe acercar u orientar parte de su investigación a un mejor conocimiento de la efectividad de la acción docente, para así conocer los puntos fuertes y los débiles y generar con ello un proceso de retroalimentación... poner, por qué no, la investigación en didáctica al servicio de la docencia...” (p.82).

Para conseguir todos estos objetivos será necesario mantener una actitud de constante reflexión y crítica, de autoperfeccionamiento, de formación, de compromiso ético con la profesión, etc.

La investigación es una tarea nuclear en el perfil del profesor universitario; esta función investigadora está muy considerada en la actualidad en los sistemas de acreditación y evaluación del profesorado universitario y posee una formación inicial específica que facilita la adquisición de las competencias necesarias para desarrollarla y cuya posesión acredita la capacidad investigadora en un área de conocimientos, el doctorado (LRU, artículo 31; LOU, artículo 38).

En la investigación, del mismo modo que ocurre con la docencia, podemos hablar de investigadores noveles e investigadores expertos y "como en todas las profesiones no todo sujeto está inclinado ni tiene las mismas habilidades para todas las funciones a desempeñar” (Galán, González y Román, 2012, p.144); y, a menudo, podemos escuchar de un investigador experimentado, la afirmación que los investigadores noveles "no saben hacer nada” (Carreras y Perrenoud, 2005, p.42), o expresado de una manera más formal "no tiene las competencias necesarias para desarrollar una investigación”.

Así, debemos considerar la necesidad de establecer una carrera profesional en esta función, sujeta a la adquisición y evaluación de competencias y a la presentación de resultados de investigaciones grupales y/o individuales (valorándose cada vez más y considerándose imprescindible que sea grupal, interdisciplinar, interuniversitaria, internacional, competitiva, etc.).

Son escasos los documentos que reflexionan respecto a las competencias investigadoras que debe atesorar el profesor universitario; aunque, por ejemplo, Pirela y Prieto (2006, p.164) hacen un listado de competencias técnicas que hacen referencia al uso, conocimiento y habilidades imprescindibles para poder diseñar y desarrollar proyectos de investigación:

- “identificar, plantear y formular problemas,

- diseñar objetivos,

- manejo de fuentes de información,

- elaboración del marco teórico,

- formular hipótesis,

- definir conceptual y operacionalmente las variables, 
- definir el tipo y diseño de investigación,

- determinar la población, el muestreo, el tamaño de la muestra,

- diseñar los instrumentos de medición,

- codificar y tabular los datos,

- seleccionar el tipo de análisis a aplicar,

- presentar, interpretar los resultados,

- elaborar el informe,

- y dominio del idioma inglés”.

Algunas competencias referidas a esta función investigadora, y que nosotros tomamos como referente en nuestro marco aplicado (a partir de Mas, 2009; Mas y Tejada, 2013; Ruiz, Mas et al. 2008), son:

\section{Función Investigadora}

\begin{tabular}{|c|c|}
\hline $\begin{array}{c}\text { Competencias } \\
\text { Investigadoras }(\mathrm{CI})\end{array}$ & Unidades de Competencia (UC) \\
\hline \multirow{14}{*}{$\begin{array}{l}\text { 1. Diseñar, desarrollar } \\
\text { y/o evaluar proyectos } \\
\text { de investigación e } \\
\text { innovación de } \\
\text { relevancia para la } \\
\text { docencia, para la } \\
\text { institución y/o para el } \\
\text { propio avance } \\
\text { científico de su área } \\
\text { de conocimiento. }\end{array}$} & $\begin{array}{l}\text { 1.1. Dominar las formas y procesos burocráticos para la concesión de ayudas } \\
\text { públicas, proyectos competitivos,... }\end{array}$ \\
\hline & $\begin{array}{l}\text { 1.2. Enmarcar las actividades investigadoras en programas, temas } \\
\text { prioritarios,... de la propia universidad, Estado, UE,... }\end{array}$ \\
\hline & 1.3. Establecer las directrices básicas de los procesos de investigación \\
\hline & $\begin{array}{l}\text { 1.4. Diseñar proyectos de investigación basados en el rigor y sistematicidad } \\
\text { científica }\end{array}$ \\
\hline & $\begin{array}{l}\text { 1.5. Participar y promover la participación/colaboración en/con diferentes } \\
\text { equipos de investigación nacionales y/o internacionales }\end{array}$ \\
\hline & $\begin{array}{l}\text { 1.6. Contribuir al establecimiento de las condiciones indispensables para } \\
\text { desarrollar actividades investigadoras }\end{array}$ \\
\hline & 1.7. Planificar colaborativamente los temas y estrategias de investigación \\
\hline & 1.8. Potenciar el/los grupo/s de investigación \\
\hline & 1.9. Estimular la reflexión compartida de los temas de investigación \\
\hline & 1.10. Asesorar investigaciones (tesinas, tesis u otros proyectos) \\
\hline & 1.11. Generar modelos teóricos de situaciones concretas de la realidad \\
\hline & $\begin{array}{l}\text { 1.12. Elaborar informes y documentos técnicos para documentar las } \\
\text { investigaciones }\end{array}$ \\
\hline & $\begin{array}{l}\text { 1.13. Promover la evaluación y la mejora constante del proceso de innovación } \\
\text { e investigación }\end{array}$ \\
\hline & $\begin{array}{l}\text { 1.14. Autodiagnosticar necesidades de formación para la mejora de la } \\
\text { competencia investigadora }\end{array}$ \\
\hline \multirow{3}{*}{$\begin{array}{l}\text { 2. Organización y } \\
\text { gestión de reuniones } \\
\text { científicas,... que } \\
\text { propicien la difusión, }\end{array}$} & 2.1. Propiciar la participación de los colaboradores/compañeros \\
\hline & 2.2. Participar en la gestión de cursos, congresos, seminarios,... \\
\hline & $\begin{array}{l}\text { 2.3. Promover y participar en grupos de trabajo respecto conocimientos del } \\
\text { área }\end{array}$ \\
\hline
\end{tabular}




\begin{tabular}{|c|c|}
\hline \multirow{3}{*}{$\begin{array}{l}\text { la comunicación, la } \\
\text { discusión, el } \\
\text { intercambio del } \\
\text { conocimiento } \\
\text { científico, la propia } \\
\text { formación }\end{array}$} & 2.4. Promover la realización de actividades inter e intrainstitucionales \\
\hline & 2.5. Participar en grupos de trabajo multidisciplinares internos y externos \\
\hline & $\begin{array}{l}\text { 2.6. Propiciar oportunidades para el intercambio de experiencias, } \\
\text { conocimientos,... }\end{array}$ \\
\hline \multirow{5}{*}{$\begin{array}{l}\text { 3. Elaborar material } \\
\text { científico actual y } \\
\text { relevante para la } \\
\text { docencia, para la } \\
\text { institución y para el } \\
\text { propio avance } \\
\text { científico de su área } \\
\text { de conocimiento }\end{array}$} & 3.1. Generar producción científica de documentos orientada a la publicación \\
\hline & 3.2. Potenciar la participación y colaboración de los compañeros/colaboradores \\
\hline & 3.3. Integrar en el trabajo propio aportaciones de terceras personas \\
\hline & 3.4. Solicitar el asesoramiento de compañeros con mayor experiencia \\
\hline & 3.5. Adaptar las ideas, producciones,... en beneficio del desarrollo grupal \\
\hline \multirow{5}{*}{$\begin{array}{l}\text { 4. Comunicar y } \\
\text { difundir } \\
\text { conocimientos, } \\
\text { avances científicos, } \\
\text { resultados de } \\
\text { proyectos de } \\
\text { investigación e } \\
\text { innovación,... a nivel } \\
\text { nacional e } \\
\text { internacional }\end{array}$} & $\begin{array}{l}\text { 4.1. Desarrollar un programa de difusión múltiple de la actividad investigadora } \\
\text { y de las producciones generados }\end{array}$ \\
\hline & 4.2. Participar en congresos para difundir el conocimiento generado \\
\hline & $\begin{array}{l}\text { 4.3. Publicar en editoriales, nacionales e internacionales, de prestigio } \\
\text { reconocido }\end{array}$ \\
\hline & 4.4. Publicar en revistas, nacionales e internacionales, de prestigio reconocido \\
\hline & 4.5. Adecuar el discurso en función del destinatario \\
\hline
\end{tabular}

Tabla I. Las 4 competencias de la función investigadora y sus 30 unidades competenciales

\section{Diseño y metodología}

El objetivo de la investigación marco consistía en delimitar el perfil competencial del profesor universitario y apuntar las implicaciones formativas que ello conlleva. Por razones de espacio, únicamente abordaremos los datos obtenidos desde el propio profesor universitario, de las 4 CI delimitadas y de las UC más significativas de las 30 establecidas.

La investigación se decantó hacia un estudio descriptivo, donde se combinaron metodologías cuantitativas y cualitativas. Se empleó una doble triangulación de agentes informantes y técnicas (Jiménez y Tejada, 2007), de este modo la información se obtuvo utilizando diferentes técnicas/intrumentos (cuestionario, entrevista y grupo de discusión) y a partir de diversas fuentes de información (expertos, profesores y alumnos), contrastando de este modo los datos obtenidos y minimizando las limitaciones de cada uno de los instrumentos.

La población objeto de estudio queda definida por el conjunto de profesores de las universidades públicas catalanas, afectados por la implantación del EEES y por las nuevas exigencias sociales, laborales y académicas que conlleva. 
La elaboración del perfil competencial, la construcción de los instrumentos y la obtención de la información se desarrolló del siguiente modo:

- Iniciamos este proceso investigativo con la revisión documental, necesaria para resumir y sintetizar la información existente relacionada con el tema de investigación, para ayudarnos a definir y seleccionar el problema, para situar los resultados del estudio en una perspectiva histórica y contextualizada, para evitar replicaciones innecesarias de investigaciones ya realizadas, para conectar nuestros hallazgos con conocimientos previos existentes, en definitiva, para la construcción del marco teórico y para seleccionar los métodos y técnicas más adecuados para desarrollar el marco aplicado.

Una vez realizada una revisión documental suficiente para centrar el tema y desde la concepción del propio investigador de lo que debe ser un profesor universitario, se elabora un primer listado provisional de competencias y unidades competenciales necesarias para la acción profesional de dicho profesional (teniendo siempre como referente las funciones de docencia e investigación que desarrolla).

- A continuación se solicitó la colaboración del grupo de investigación CIFO de la Universidad Autónoma de Barcelona (por la larga experiencia investigadora de sus miembros en temas próximos conceptualmente a esta investigación), con la intención de organizar un grupo de discusión de expertos (técnica de trabajo en grupo). El objetivo de organizar dicho grupo de discusión era establecer un foro de discusión académico generador de un intercambio de ideas, opiniones, conocimientos,... para depurar y ajustar el listado provisional de competencias profesionales del profesor universitario realizado y, de este modo, avanzar hacia la delimitación del perfil competencial necesario para que el profesor universitario desarrolle correctamente las funciones que se le están solicitando en el nuevo contexto y modelo universitario hacia el que estamos avanzando en la actualidad.

- Organizamos un grupo de discusión (con la incorporación de algunos elementos de la técnica Delphi) con los expertos anteriormente mencionados; se organizaron tres sesiones (con una duración comprendida entre 45 y 75 minutos) en las que el autor de este trabajo actuaba como moderador, facilitándose previamente a los expertos participantes el listado de competencias y unidades competenciales (realizado a partir de la revisión documental), su fundamentación teórica y, cuando procedió, las aportaciones realizadas en las sesiones anteriores. En cada sesión, estos expertos, ofrecían un feedback del material que se les había facilitado anteriormente y que ellos habían analizado (propuestas de modificación, de eliminación, de incorporación,...), sometiéndose a intensas discusiones en el seno del grupo de discusión y siendo estas valiosas aportaciones nuevamente sintetizadas e incorporadas al perfil profesional que estaba en construcción; obteniéndose, al finalizar este proceso cíclico (esta acción de entrega-devolución recibe el nombre de circulación), un segundo listado provisional (contrastado con expertos-profesores universitarios) de competencias y de unidades competenciales asociadas a la acción profesional del profesor universitario, discriminándose las pertenecientes a la función investigadora y las correspondientes a la función docente. 
- Este último listado de competencias y de unidades competenciales fue sometido a la validación de 11 jueces expertos en la temática (empleando los criterios de univocidad, pertinencia, importancia), incorporando las propuestas más coincidentes y significativas, obteniendo finalmente el perfil competencial del profesor universitario en su función docente e investigadora. Después de recibir las validaciones efectuadas por los expertos se procedió al análisis cuantitativo del cuestionario, obteniendo un Alpha de Cronbach de 0,97 para el conjunto de las competencias, de 0,97 para las competencias referentes a docencia y de 0,956 para las referentes a investigación)

- A partir de la obtención de este perfil competencial, en lo referente a los expertos utilizamos una unidad muestral intencional no probabilística, conformada por personas relevantes en la temática que nos atañe, bien por su desempeño profesional (responsables de formación del profesorado universitario, investigadores,...) o por su situación privilegiada como gestores académicos (vicedecanos y vicerrectores de investigación y docencia, directores de departamento, etc.), pudiendo aportar, mediante entrevistas en profundidad, una visión y datos difícilmente obtenibles mediante otros informantes.

- En lo referente al profesorado, utilizamos un sistema de muestreo aleatorio por conglomerados (institución universitaria); optando por delimitar la población a las universidades públicas catalanas. El profesorado participó en la investigación cumplimentando un cuestionario donde se consideraban variables personales, formativas y sociolaborales-contextuales y variables respecto al perfil competencial del docente e investigador. A continuación, a modo de ejemplo y para facilitar la posterior comprensión de los resultados, se presenta la estructura de los ítems donde se solicitaba la valoración de las unidades competenciales que componen cada competencia y su escala (comprendida entre 1 [valoración mínima] y 5 [valoración máxima] según el grado de posesión y de necesidad de dominio):

\begin{tabular}{|c|c|c|c|c|c|c|c|c|c|c|}
\hline \multirow{2}{*}{$\begin{array}{l}\text { CI 1: Diseñar, desarrollar y/o evaluar proyectos de } \\
\text { investigación e innovación de relevancia para la } \\
\text { docencia, para la institución y/o para el propio avance } \\
\text { científico de su área de conocimiento. }\end{array}$} & \multicolumn{5}{|c|}{ Dominio Actual } & \multicolumn{5}{|c|}{$\begin{array}{c}\text { Necesidad de } \\
\text { dominio }\end{array}$} \\
\hline & 1 & 2 & 3 & 4 & 5 & 1 & 2 & 3 & 4 & 5 \\
\hline \multicolumn{11}{|l|}{ UCI. 1.1. ... } \\
\hline UCI. 1.2. ... & & & & & & & & & & \\
\hline
\end{tabular}

Tabla II. Ejemplo de la estructura de los ítems del cuestionario.

- Los alumnos implicados cursaban los últimos cursos de licenciaturas o estudios de postgrado del ámbito de Ciencias de la Educación, resultando sus aportaciones especialmente útiles respecto la competencias que posee y/o debería poseer un profesor universitario, ya que poseen la percepción propia de un alumno enriquecida por los conocimientos y criterios psicopedagógicos que atesoran. Dichos alumnos cumplimentaron un cuestionario idéntico al del profesorado respecto al perfil, aunque contextualizado a su situación en el resto de apartados. 
Finalmente la muestra de la investigación se configuró del siguiente modo:

\begin{tabular}{ccccc}
\hline $\begin{array}{c}\text { Universidades } \\
\text { Participantes }\end{array}$ & $\begin{array}{c}\text { Profesorado } \\
\text { participante }\end{array}$ & $\begin{array}{c}\text { Alumnos } \\
\text { participantes }\end{array}$ & $\begin{array}{c}\text { Expertos } \\
\text { entrevistados }\end{array}$ & $\begin{array}{c}\text { Expertos } \\
\text { participantes en } \\
\text { grupos discusión }\end{array}$ \\
\hline 6 & 161 & 63 & 11 & 7 \\
\hline
\end{tabular}

Tabla III. Muestra implicada

Caracterización de la muestra

En la tabla que presentamos a continuación podemos observar una síntesis de la caracterización de la muestra de profesorado participante en la investigación.

\begin{tabular}{|c|c|}
\hline Edad & 42,96 años \\
\hline \multirow{2}{*}{ Género } & Masculino: 53,20\% \\
\hline & Femenino: 46,80\% \\
\hline \multirow{3}{*}{ Titulación } & Doctorado: 77,50\% \\
\hline & Licenciado: 20,60\% \\
\hline & Diplomado: 1,90\% \\
\hline $\begin{array}{l}\text { Experiencia docente } \\
\text { no universitaria }\end{array}$ & $\begin{array}{l}39,2 \% \\
\text { con una experiencia media de } 5,4 \text { años }\end{array}$ \\
\hline $\begin{array}{l}\text { Experiencia docente } \\
\text { universitaria }\end{array}$ & $\begin{array}{l}14 \text { años de media } \\
\text { (mínima 1,5 años - máxima } 38 \text { años) }\end{array}$ \\
\hline $\begin{array}{l}\text { Experiencia } \\
\text { investigadora }\end{array}$ & $\begin{array}{l}\text { Grupal: } 7,8 \text { años de media } \\
\text { Individual: } 4,7 \text { años de media }\end{array}$ \\
\hline $\begin{array}{l}\text { Ámbitos de } \\
\text { conocimiento }\end{array}$ & $\begin{array}{l}\text { CC de la Educación: } 32,7 \% \\
\text { CC Sociales y Humanas: } 42,1 \% \\
\text { CC de la Salud, Experimentales e Ingeniería: } 25,1 \%\end{array}$ \\
\hline ANTIGÜEDAD & $\begin{array}{l}\text { Profesorado novel ( }<5 \text { años): } 24,2 \% \\
\text { Profesorado experimentado ( }>5 \text { años): } 75,8 \%\end{array}$ \\
\hline $\begin{array}{l}\text { Situación } \\
\text { Contractual }\end{array}$ & $\begin{array}{l}\text { Profesorado consolidado: } 51,25 \% \\
\text { Profesorado temporal: } 19,37 \% \\
\text { Profesorado asociado: } 29,37 \%\end{array}$ \\
\hline Dedicación & $\begin{array}{l}\text { Tiempo completo: } 72,6 \% \\
\text { Tiempo parcial: } 27,4 \%\end{array}$ \\
\hline
\end{tabular}

\section{Tabla IV. Caracterización de la muestra}

Otros datos interesantes para conocer más el perfil del profesor universitario en la muestra analizada, desde la perspectiva de la antigüedad, son los siguientes: en primer lugar, en el caso del profesorado novel (media de edad 35,14 años) un $40 \%$ son doctores, mientras que en el caso del profesorado experimentado (media de edad 45,32 años) este porcentaje alcanza el $89,6 \%$; en segundo lugar, la antigüedad del profesorado en la institución está muy relacionada con la situación contractual del 
mismo, el profesorado consolidado está compuesto por un 2,6\% de profesorado novel y un $97,4 \%$ de profesorado experimentado, en cambio el profesorado no consolidado está conformado por un $30 \%$ de profesorado novel y por un $70 \%$ de profesorado experimentado.

\section{Resultado}

En este artículo indagaremos sobre la percepción del dominio competencial del profesor novel ( $<5$ años de experiencia en la institución) y del profesor experimentado ( $>5$ años de experiencia en la institución) y conoceremos sus respectivas valoraciones sobre las competencias investigadoras más necesarias para desarrollar sus funciones adecuadamente en el nuevo contexto que se está configurando.

Consideraremos las competencias como eje vertebrador y como elementos aglutinadores de las UC en que se basó nuestra investigación, aunque en determinados apartados deberemos hacer referencia a estas últimas para desgranar diferentes significados y valoraciones. En el cuerpo principal del documento únicamente se presenta la tabla $\mathrm{V}$ con los resultados globales de las CI, para más información respecto a los resultados obtenidos de las UC que las componen dirigirse al anexo de la publicación y ver las tablas VII, VIII, IX y X.

Dominio actual y necesidad de dominio de las competencias investigadoras según la experiencia

Si atendemos a los resultados obtenidos en cada una de las CI delimitadas (media aritmética de las UC que la integran), no existe diferencia estadística significativa $(\operatorname{sig}<0,05)$ entre las valoraciones que realizan los profesores noveles y experimentados respecto a su percepción de dominio competencial necesario, pero si presentan diferencias estadísticamente significativas en el dominio actual de las CI.

\begin{tabular}{|c|c|c|c|c|c|c|}
\hline \multirow{2}{*}{$\begin{array}{l}\text { Competencias } \\
\text { Investigadoras }\end{array}$} & \multicolumn{3}{|c|}{$\begin{array}{c}\text { Dominio actual } \\
\text { según experiencia }\end{array}$} & \multicolumn{3}{|c|}{$\begin{array}{l}\text { Necesidad dominio } \\
\text { según experiencia }\end{array}$} \\
\hline & Novel & $\begin{array}{l}\text { Experime } \\
\text { ntado }\end{array}$ & Sig & Novel & $\begin{array}{l}\text { Experime } \\
\text { ntado }\end{array}$ & Sig \\
\hline $\begin{array}{l}\text { CI1: Diseñar, desarrollar y/o } \\
\text { evaluar proyectos de } \\
\text { investigación e innovación de } \\
\text { relevancia para la docencia, para } \\
\text { la institución y/o para el propio } \\
\text { avance científico de su área de } \\
\text { conocimiento. }\end{array}$ & 2,69 & 3,11 & $\begin{array}{l}, 009 \\
1<2\end{array}$ & 4,16 & 4,27 & - \\
\hline $\begin{array}{l}\text { CI2: Organizar y gestionar } \\
\text { reuniones, seminarios, jornadas, }\end{array}$ & 2,81 & 3,21 & ,034 & 4,25 & 4,22 & - \\
\hline
\end{tabular}




\begin{tabular}{l}
\hline congresos científicos,... que \\
propicien la difusión, la \\
comunicación, la discusión, la \\
reflexión, el intercambio del \\
conocimiento científico y de sus \\
progresos, así como la propia \\
formación docente e \\
investigadora
\end{tabular}

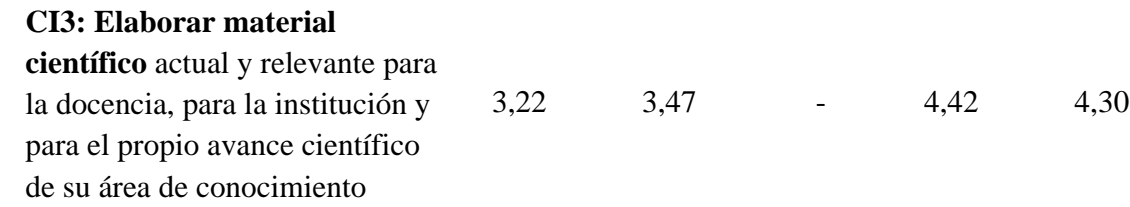

\section{CI4: Comunicar y difundir}

conocimientos, avances

científicos, resultados de

proyectos de investigación e

$\begin{array}{lllll}2,74 & 3,27 & , 002 & & \\ & & 1<2 & 4,27 & 4,34\end{array}$

innovación,... a nivel nacional e

internacional

Tabla V. Valoración del dominio actual y necesidad de dominio de las CI, según la experiencia.

Los valores que sustentan el perfil competencial investigador actual del profesor experimentado son superiores que los del profesor novel. A continuación procederemos a comentar únicamente las CI relativas al dominio actual, que son las que ofrecen diferencias estadísticamente significativas, y algunas de sus UC que también presentan estas diferencias significativas.

1. Los resultados obtenidos por el profesorado experimentado y el novel en el dominio actual de las UC de la CI1 "diseñar, desarrollar y/o evaluar proyectos de investigación e innovación” nos indican un menor dominio del profesorado novel, mostrando diferencias significativas estadísticamente: la UC1.1 “dominar las formas y procesos administrativos para la solicitud-adjudicación de ayudas públicas, privadas, proyectos competitivos,...", la UC1.5 "participar y promover la participación/colaboración en/con diferentes equipos de investigación nacionales y/o internacionales", la UC1.6 "contribuir al establecimiento de las condiciones indispensables para desarrollar actividades investigadoras” y la UC1.10, “asesorar investigaciones...”.

Dicho profesorado novel manifiesta tener un dominio competencial por debajo de la media teórica en todas las UC, exceptuando la UC1.4, "diseñar proyectos de investigación basados en el rigor y sistematicidad científica” y la UC1.12, "elaborar informes y documentos técnicos para documentar las investigaciones" que están levemente por encima la media teórica $(3,11)$. Este bajo dominio 
competencial es razonable considerando la etapa inicial de desarrollo profesional en la que se encuentra el colectivo.

Un aspecto preocupante a destacar es el bajo dominio que admite tener el profesorado experimentado en todas las UC presentadas. Se sitúan por debajo de la media teórica (5 de 14 presentadas) la UC1.1 “dominar las formas y procesos administrativos para la solicitud-adjudicación de ayudas públicas, privadas, proyectos competitivos,...", la UC1.2. "enmarcar las actividades investigadoras en programas, temas prioritarios,...”, la UC1.3 “establecer las directrices básicas de los procesos de investigación (plan estratégico)", la UC1.11 "generar modelos teóricos de situaciones concretas de la realidad" y la UC1.13 "promover la evaluación y la constante mejora del proceso de innovación e investigación”; el resto (3) superan, aunque levemente, la media teórica.

2. En segundo lugar, si se comparan los resultados obtenidos del dominio actual expresado por el profesorado experimentado y novel, en las UC de la CI2 “Organizar y gestionar reuniones, seminarios, jornadas, congresos científicos,...”, cabe resaltar que los valores que sustentan el perfil competencial del profesor experimentado son ligeramente superiores que los del profesor novel (todos ellos por debajo de la media teórica) observándose diferencias significativas en las UC2.1 "propiciar la participación de los colaboradores/compañeros", UC2.2 "participar en la gestión de cursos, congresos, seminarios...", UC2.5 "participar en grupos de trabajo multidisciplinares internos y externos", UC2.6 "propiciar oportunidades para el intercambio de experiencias, conocimientos,...”; y, aunque no se obtienen diferencias significativas, la UC2.4 "promover la realización de actividades inter $e$ intrainstitucionales" obtiene una puntuación inferior a la media en ambos colectivos.

3. Comparando los resultados obtenidos del dominio actual expresado, por el profesorado experimentado y el novel, en las UC relacionadas con la CI3 "elaboración de material científico", únicamente muestra una diferencia con significatividad estadística el resultado asociado a la UC3.2 "potenciar la participación y colaboración de los compañeros/colaboradores”, donde el profesor novel obtiene una puntuación menor a la media, al igual que ocurre con la UC3.1 "generar producción científica de documentos orientada a la publicación”.

4. El dominio actual expresado por el profesorado experimentado de las UC correspondientes a la CI4 “comunicación y difusión de conocimientos...", lógicamente son mayores que los valores presentados por el profesor novel.

Los resultados de las tres UC que arrojan significatividad estadística y que también obtienen una puntuación inferior a la media en el profesorado novel son la UC4.2 "participar en congresos para difundir el conocimiento generado", la UC4.3 "publicar en editoriales, nacionales e internacionales, de impacto y prestigio reconocido" y, por último, la UC4.4 "publicar en revistas, nacionales $e$ internacionles, de impacto y prestigio reconocido". Del mismo modo obtiene un 
resultado inferior a la media y la mínima valoración en los 2 colectivos la UC4.1 "desarrollar un programa de difusión múltiple de la actividad investigadora y de las producciones generadas", aspecto preocupante y motivo de reflexión especialmente en el profesorado experimentado.

Aunque inicialmente nos comprometimos a presentar únicamente los datos obtenidos del propio profesor universitario, no podemos concluir este apartado sin apuntar la opinión de los expertos entrevistados.

En la tabla que presentamos a continuación mostramos la priorización de las competencias investigadoras realizada por los mencionados expertos (desde los criterios de importancia y de necesidad formativa), diferenciando entre profesorado novel y experimentado:

\begin{tabular}{|c|c|c|}
\hline \multicolumn{3}{|c|}{ Necesidad dominio } \\
\hline \multicolumn{2}{|c|}{ Priorización de Expertos } & \multirow[b]{2}{*}{ Competencias Investigadoras } \\
\hline $\begin{array}{c}\text { Profesor } \\
\text { Novel }\end{array}$ & $\begin{array}{c}\text { Profesor } \\
\text { Experimentado }\end{array}$ & \\
\hline $1^{\mathrm{a}}$ & $1^{\mathrm{a}}-2^{\mathrm{a}}$ & $\begin{array}{l}\text { CI1: Diseñar, desarrollar y/o evaluar proyectos de investigación e } \\
\text { innovación de relevancia para la docencia, para la institución y/o } \\
\text { para el propio avance científico de su área de conocimiento. }\end{array}$ \\
\hline $3^{a}-4^{a}$ & $3^{a}$ & $\begin{array}{l}\text { CI2: Organizar y gestionar reuniones, seminarios, jornadas, } \\
\text { congresos científicos,... que propicien la difusión, la comunicación, } \\
\text { la discusión, la reflexión, el intercambio del conocimiento científico } \\
\text { y de sus progresos, así como la propia formación docente e } \\
\text { investigadora }\end{array}$ \\
\hline $2^{\mathrm{a}}$ & $4^{\mathrm{a}}$ & $\begin{array}{l}\text { CI3: Elaborar material científico actual y relevante para la docencia, } \\
\text { para la institución y para el propio avance científico de su área de } \\
\text { conocimiento }\end{array}$ \\
\hline $3^{a}-4^{a}$ & $\mathbf{1}^{\mathrm{a}}-2^{\mathrm{a}}$ & $\begin{array}{l}\text { CI4: Comunicar y difundir conocimientos, avances científicos, } \\
\text { resultados de proyectos de investigación e innovación,... a nivel } \\
\text { nacional e internacional }\end{array}$ \\
\hline
\end{tabular}

Tabla VI. Priorización (según los expertos entrevistados) del dominio necesario de las CI según la antigüedad del profesor universitario.

En lo referente a las CI del colectivo novel, los expertos priorizan una necesidad formativa en la CI1, "diseñar, desarrollar y/o evaluar proyectos de investigación $e$ innovación de relevancia para la docencia, para la institución y/o para el propio avance científico de su área de conocimiento", y en la CI3, "elaborar material científico actual y relevante para la docencia, para la institución y para el propio avance científico de su área de conocimiento"; en cambio el profesorado novel (ver tabla V) pasa a priorizar en primer lugar, en necesidad de dominio, la CI3,"elaborar material científico actual y relevante para la docencia, para la institución y para el propio avance científico de su área de conocimiento", y la CI4, “comunicar y difundir conocimientos, avances científicos, resultados de proyectos de investigación e innovación,... a nivel nacional e internacional", dejando como menor necesidad de dominio la CI1 "diseñar, desarrollar y/o evaluar proyectos de investigación e innovación de relevancia para la docencia, para la institución y/o para el propio avance científico de su área de conocimiento”. 
Esta diferencia entre los resultados de los expertos y del propio profesorado novel puede estar influenciado por los criterios establecidos en los sistemas de acreditación, donde se valora principalmente la publicación de resultados científicos, pero resulta evidente que para poder difundir resultados en primer lugar se deben diseñar y/o desarrollar proyectos de investigación.

En lo referente a las CI del colectivo experimentado, existe gran coincidencia entre las propuestas de los expertos y la del propio profesorado experimentado, ambos colectivos priorizan como competencias con mayor necesidad de dominio la CI3 "elaborar material científico actual y relevante para la docencia, para la institución y para el propio avance científico de su área de conocimiento" y la CI4 "comunicar y difundir conocimientos, avances científicos, resultados de proyectos de investigación e innovación,... a nivel nacional e internacional”; partiendo los expertos del supuesto que el profesorado experimentado ya ha adquirido las CI1 y CI2 mediante su formación doctoral, la propia práctica y su trayectoria profesional.

\section{A modo de conclusión y de recomendaciones}

El profesor universitario doctor, es un investigador de máximo nivel y con una alta cualificación, pero antes de llegar a este nivel de desarrollo profesional debe pasar por unos estadios previos para adquirir y/o desarrollar las competencias necesarias.

Como primera recomendación consideramos necesario establecer una trayectoria, un itinerario, para facilitar un desarrollo profesional claro en la función investigadora del perfil profesional del profesor universitario. Para ello consideramos irrenunciable un proceso de socialización, evidentemente más guiado en su etapa inicial de incorporación, contextualizado según sea el ámbito científico en el que esté inmerso el investigador; pudiéndose desarrollar este proceso en un grupo de investigación, en un laboratorio, etc.

En este proceso puede responsabilizarse de diferentes tareas, de diversa tipología y dificultad, que integran un proceso de investigación: búsqueda de información y consultas documentales, realización de observaciones y entrevistas, pasación de cuestionarios, codificación, introducción y análisis de datos, elaboración de preinformes, etc.; siéndole ello útil para la adquisición y desarrollo de sus competencias investigadoras y para contribuir a la mencionada socialización profesional e institucional del investigador novel.

Como segunda recomendación, a partir de los resultados obtenidos, consideramos lógico recomendar al profesorado cursar diferentes acciones formativas según su experiencia; coincidiendo con los resultados de la investigación efectuada por Mayor (1996), que indica que deben ser diferenciadas según su situación profesional, contexto, características de cada especialidad, debiendo partir de los problemas concretos y reales del profesorado.

Así, integrando los resultados obtenidos del propio profesor (tablas V, VII, VIII, IX y X) y de los expertos (tabla VI), proponemos priorizar áreas de formación y agrupar áreas temáticas para desarrollar las competencias asociadas a la función investigadora: 
1. Al profesorado novel debería ofrecérsele módulos formativos referentes a "diseño, desarrollo y evaluación de proyectos de investigación e innovación”, "organización y gestión de eventos científicos” e, intentando agrupar el resto de UC valoradas por debajo de la media, una acción formativa respecto a "generar y difundir producción científica"; añadiendo como aspecto transversal en dichas acciones formativas "el trabajo colaborativo". Aunque resulta curioso, coincidiendo con las aportaciones realizadas por los expertos en docencia universitaria en la investigación de Torra (2010) cuando se les pregunta por propuesta concretas de formación, que uno de los aspectos reiterados en el perfil competencial presentado sea la "participación”, la “colaboración”, el "trabajo en equipo" y que académicamente se valoren los proyectos colaborativos entre compañeros, grupos de investigación, universidades, estados, etc;. En cambio, como explicitan Galán, González y Román (2012, citando a Ortega, 2003), la realidad de nuestro sistema universitario, en cuanto a promoción y méritos se refiere, propicia la individualidad y la competencia entre compañeros, grupos, etc.

2. Al profesorado experimentado debería ofrecérsele módulos formativos referentes a la CI3 "elaborar material científico actual y relevante para la docencia, para la institución y para el propio avance científico de su área de conocimiento", en concreto al "establecimiento de planes estratégicos de difusión múltiple de la producción científica", y a la CI4 "comunicar y difundir conocimientos, avances científicos, resultados de proyectos de investigación e innovación,... a nivel nacional e internacional”; aunque sin descartar elementos de la CI1 como "establecimiento de líneas de trabajo y aspectos burocrático-administrativos de gestión de proyectos para la participación en convocatorias competitivas" y "elaboración de protocolos de evaluación y mejora continua en los procesos de investigación e innovación".

Esta formación debería facilitar la actualización y especialización investigadora del profesorado, pudiendo adoptar diversas tipologías (cursos, seminarios, grupos de trabajo/discusión específicos, tertulias, mentorización, coaching...) y modalidades (presencial, semipresencial y a distancia) según las características y necesidades del profesorado e institución, según la finalidad concreta de la acción formativa, etc.

Es difícil contrastar los resultados obtenidos en esta investigación con otros publicados recientemente, ya que habitualmente los estudios existentes se centran en delimitar el perfil competencial de la función docente y/o sus necesidades formativas (Álvarez Rojo et al, 2009, 2009b, 2011; Galán, González y Román, 2012; Torra, 2010), no haciendo referencia al perfil competencial investigador ni a sus necesidades formativas. 


\section{Referencias bibliográficas}

ALVAREZ ROJO, V. et al. (2009). Perfiles y competencias docentes requeridos en el contexto actual de la educación universitaria. Revista Española de Orientación y Psicopedagia, 20 (3), 270-283.

ALVAREZ ROJO, V. et al. (2009b). Perfiles docentes para el Espacio Europeo de Educación Superior (EEES) en el ámbito universitario español. Revista Electrónica de Investigación y Evaluación Educativa, 15 (1), 1-18.

ALVAREZ ROJO, V. et al. (2011). Necesidades de formación del profesorado universitario para la adpatación de su docencia al Espacio Europeo de Educación Superior (EEES). Revista Electrónica de Investigación y Evaluación Educativa, 17 (1), 1-22.

GALÁN, A., GONZÁLEZ, M.A. Y ROMÁN, M. (2012). La irrupción del factor comunitario en el perfil del profesor universitario. Bordon, 64 (3), 133-148.

GONZÁLEZ SOTO, A. P. (2005). Posibilidades de formación en el Espacio Europeo de Educación Superior. En J. CABERO (coord.), Formación del Profesorado universitario para la incorporación del aprendizaje en red en el EEES. Sevilla: Universidad de Sevilla [soporte CD].

ICE-Universidad de Zaragoza (2004). Programa de mejora e innovación de la docencia en el marco de la convergencia al EEES. Zaragoza: Universidad de Zaragoza.

IGLESIA, C. (2011). Adecuación del grado de desarrollo de la formación en competencias a la necesidad en el entorno laboral según la opinión de los estudiantes. Revista Complutense de Educación, 22 (1), 71-92. http://revistas.ucm.es/index.php/RCED/issue/view/2111/showToc (Consultado: 5 de enero de 2014].

JIMÉNEZ JIMÉNEZ, B. y TEJADA, J. (2007). Procesos y métodos de investigación, en J. Tejada y V. Giménez (Coords.), Formación de formadores. Escenario Institucional, 543-629. Madrid: ITES-Paraninfo.

LEY ORGÁNICA 11/1983, de 25 de agosto, de Reforma Universitaria.

LEY ORGÁNICA 6/2001, de 21 de diciembre, de Universidades.

MAS, O. (2009). El perfil competencial del profesorado universitario en el Espacio Europeo de Educación Superior (Tesis Doctoral inédita). Bellaterra: Universidad Autónoma de Barcelona.

MAS, O (2011). El profesor universitario: sus competencias y formación. Profesorado. Revista de curriculum y formación del profesorado, 15 (3), 1-17. http://www.ugr.es/ recfpro/?p=958 (Consultado: 10 de enero de 2014].

MAS, O. (2012). Las competencias del docente universitario: la percepción del alumno, de los expertos y del propio protagonista. Revista de Docencia 
Universitaria. REDU, 10 (2), 299-318. http://red-u.net/redu/index.php/REDU/issue/ view/67/showToc (Consultado: 29 de diciembre de 2013).

MAS, O. y OLMOS, P. (2011). Competencias docentes para la inclusión del alumnado universitario en el marco del Espacio Europeo de Educación Superior. Revista de educación inclusiva, vol. 5(1), 159-174.

MAS, O. y TEJADA, J. (2013). Funciones y competencias en la docencia universitaria. Madrid: Sintesis.

MAYOR, C. (1996). Las funciones del profesor universitario analizadas por sus protagonistas. Un estudio atendiendo al grupo de titulación y los años de experiencia, en la Universidad de Sevilla. Relieve, vol.2(1) http://www.uv.es/RELIEVE/v2n1/RELIEVEv2n1.htm (Consultado: 30 de diciembre de 2013).

RUIZ, C.; MAS, O. et al (2008). Funciones y escenarios de actuación del profesor universitario. Apuntes para la definición del perfil basado en competencias. Revista de la Educación Superior, XXXVII(2) (146), 115-132.

TEJADA, J. (2005). La formación profesional superior y el EEES, en J. Tejada et al., Actas IV Congreso de formación para el Trabajo. Nuevos escenarios de trabajo y nuevos retos en la formación. Madrid: Tornapunta Ediciones.

TOMÀS, M. (2001). Presentación. Educar, 28, 6-9.

TOMÀS, M; CASTRO, D. y FEIXAS, M. (2012). Tensiones entre las funciones docente e investigadora del profesorado en la universidad. Revista de docencia $\begin{array}{lllll}\text { universitaria. } & R E D U, & 10 & \text { (1), 343-367. } & \text { http://red- }\end{array}$ u.net/redu/index.php/REDU/issue/view/66/showToc (Consultado: 28 de diciembre de 2013).

TOMÁS, M. e ION, G. (2008). Contrastando dos modelos de análisis de la cultura organizativa de la universidad. Bordón, 60 (3), 131-147.

ZABALZA, M.A. (2012). El estudio de las "buenas prácticas" docentes en la enseñanza universitaria. Revista de Docencia Universitaria. REDU, 10 (1), 17-42. http://www.red-u.net/redu/index.php/REDU/issue/view/66/showToc (Consultado: 28 de diciembre de 2013).

\section{Referencias digitales}

CARRERAS, J. y PERRENOUD, P. (2005). El debat sobre les competències en l'ensenyament universitari. Barcelona: ICE-UB. http://diposit.ub.edu/dspace /handle/2445/1042 (Consultado: 5 de enero de 2014).

TORRA, I. (2010). Identificación, desarrollo y evaluación de competencias docentes en la aplicación de planes de formación dirigidos a profesorado universitario. http://gifd.upc.edu/?page_id=88\&lang=ca (Consultado: 4 de enero de 2013). 


\section{Correspondencia con el Autor}

Òscar MAS-TORELLÓ

Universidad Autónoma de Barcelona (UAB)

Facultad de Ciencias de la Educación.

Departamento Pedagogía Aplicada (Edificio G6 - Despacho 264).

08193-Bellaterra (Cerdanyola del Vallès).

Barcelona (España).

e-mail: oscar.mas@uab.cat 
Anexo

\begin{tabular}{|c|c|c|c|c|c|c|}
\hline \multirow{2}{*}{$\begin{array}{l}\text { CI1: Diseñar, desarrollar y/o evaluar } \\
\text { proyectos de investigación e innovación de } \\
\text { relevancia para la docencia, para la } \\
\text { institución y/o para el propio avance } \\
\text { científico de su área de conocimiento. }\end{array}$} & \multicolumn{3}{|c|}{$\begin{array}{c}\text { Dominio actual } \\
\text { según experiencia }\end{array}$} & \multicolumn{3}{|c|}{$\begin{array}{l}\text { Necesidad dominio } \\
\text { según experiencia }\end{array}$} \\
\hline & Novel & $\begin{array}{l}\text { Experimen } \\
\text { tado }\end{array}$ & Sig & Novel & $\begin{array}{l}\text { Experimen } \\
\text { tado }\end{array}$ & Sig \\
\hline $\begin{array}{l}\text { UC1.1.Dominar las formas y procesos } \\
\text { administrativos para la solicitud- } \\
\text { adjudicación de ayudas públicas, } \\
\text { privadas, proyectos competitivos,... }\end{array}$ & 2,34 & 2,96 & $\begin{array}{l}, 007 \\
1<2\end{array}$ & 4,06 & 4,29 & - \\
\hline $\begin{array}{l}\text { UC1.2.Enmarcar las actividades } \\
\text { investigadoras en programas, temas } \\
\text { prioritarios,... de la propia universidad, } \\
\text { autonomía, Estado, UE,... }\end{array}$ & 2,62 & 2,93 & - & 3,88 & 4,24 & - \\
\hline $\begin{array}{l}\text { UC1.3.Establecer las directrices básicas de } \\
\text { los procesos de investigación (plan } \\
\text { estratégico) }\end{array}$ & 2,71 & 2,87 & - & 4,17 & 4,15 & - \\
\hline $\begin{array}{l}\text { UC1.4.Diseñar proyectos de investigación } \\
\text { basados en el rigor y sistematicidad } \\
\text { científica }\end{array}$ & 3,11 & 3,50 & - & 4,54 & 4,47 & - \\
\hline $\begin{array}{l}\text { UC1.5.Participar y promover la } \\
\text { participación/colaboración en/con } \\
\text { diferentes equipos de investigación } \\
\text { nacionales y/o internacionales }\end{array}$ & 2,57 & 3,19 & $\begin{array}{l}, 005 \\
1<2\end{array}$ & 4,43 & 4,50 & - \\
\hline $\begin{array}{l}\text { UC1.6.Contribuir al establecimiento de las } \\
\text { condiciones indispensables para } \\
\text { desarrollar actividades investigadoras }\end{array}$ & 2,59 & 3,06 & $\begin{array}{l}, 033 \\
1<2\end{array}$ & 4,12 & 4,33 & - \\
\hline $\begin{array}{l}\text { UC1.7.Planificar colaborativamente los } \\
\text { temas y estrategias de investigación }\end{array}$ & 2,83 & 3,24 & - & 4,31 & 4,30 & - \\
\hline $\begin{array}{l}\text { UC1.8.Potenciar el/los grupo/s de } \\
\text { investigación }\end{array}$ & 2,85 & 3,10 & - & 4,26 & 4,32 & - \\
\hline $\begin{array}{l}\text { UC1.9.Estimular la reflexión compartida de } \\
\text { los temas de investigación }\end{array}$ & 2,76 & 3,05 & - & 4,15 & 4,37 & - \\
\hline $\begin{array}{l}\text { UC1.10.Asesorar investigaciones (tesinas, } \\
\text { tesis u otros proyectos) }\end{array}$ & 2,31 & 3,48 & $\begin{array}{l}, 000 \\
1<2\end{array}$ & 4,09 & 4,32 & - \\
\hline $\begin{array}{l}\text { UC1.11.Generar modelos teóricos de } \\
\text { situaciones concretas de la realidad }\end{array}$ & 2,61 & 2,79 & - & 3,97 & 3,87 & - \\
\hline $\begin{array}{l}\text { UC1.12.Elaborar informes y documentos } \\
\text { técnicos para documentar las } \\
\text { investigaciones }\end{array}$ & 3,11 & 3,32 & - & 4,17 & 4,16 & - \\
\hline $\begin{array}{l}\text { UC1.13.Promover la evaluación y la } \\
\text { constante mejora del proceso de } \\
\text { innovación e investigación }\end{array}$ & 2,62 & 2,94 & - & 4,24 & 4,18 & - \\
\hline $\begin{array}{l}\text { UC1.14.Autodiagnosticar necesidades de } \\
\text { formación para la mejora de la } \\
\text { competencia investigadora }\end{array}$ & 2,77 & 3,16 & - & 3,97 & 4,22 & - \\
\hline
\end{tabular}

Tabla VII. Valoración del dominio actual y de de la necesidad de dominio del diseño, desarrollo y/o evaluación de proyectos de investigación e innovación según la experiencia del profesor. 


\begin{tabular}{|c|c|c|c|c|c|c|}
\hline \multirow{2}{*}{$\begin{array}{l}\text { CI2: Organizar y gestionar reuniones, } \\
\text { seminarios, jornadas, congresos } \\
\text { científicos,... que propicien la difusión, } \\
\text { la comunicación, la discusión, la } \\
\text { reflexión, el intercambio del } \\
\text { conocimiento científico y de sus } \\
\text { progresos, así como la propia } \\
\text { formación docente e investigadora }\end{array}$} & \multicolumn{3}{|c|}{$\begin{array}{c}\text { Dominio actual } \\
\text { según experiencia }\end{array}$} & \multicolumn{3}{|c|}{$\begin{array}{l}\text { Necesidad dominio } \\
\text { según experiencia }\end{array}$} \\
\hline & Novel & $\begin{array}{l}\text { Experimen } \\
\text { tado }\end{array}$ & Sig & Novel & $\begin{array}{c}\text { Experimen } \\
\text { tado }\end{array}$ & Sig \\
\hline $\begin{array}{l}\text { UC2.1.Propiciar la participación de los } \\
\text { colaboradores/compañeros }\end{array}$ & 2,80 & 3,36 & $\begin{array}{c}0,015 \\
1<2\end{array}$ & 4,35 & 4,31 & - \\
\hline $\begin{array}{l}\text { UC2.2.Participar en la gestión de cursos, } \\
\text { congresos, seminarios,... }\end{array}$ & 2,69 & 3,29 & $\begin{array}{c}0,007 \\
1<2\end{array}$ & 4,12 & 4,09 & - \\
\hline $\begin{array}{l}\text { UC2.3.Participar en grupos de trabajo } \\
\text { respecto conocimientos del área }\end{array}$ & 3,21 & 3,25 & - & 4,36 & 4,19 & - \\
\hline $\begin{array}{l}\text { UC2.4.Promover la realización de } \\
\text { actividades inter e intrainstitucionales }\end{array}$ & 2,69 & 2,97 & - & 4,15 & 4,17 & - \\
\hline $\begin{array}{l}\text { UC2.5.Participar en grupos de trabajo } \\
\text { multidisciplinares internos y externos }\end{array}$ & 2,71 & 3,18 & $\begin{array}{l}0,05 \\
1<2\end{array}$ & 4,24 & 4,32 & - \\
\hline $\begin{array}{l}\text { UC2.6.Propiciar oportunidades para el } \\
\text { intercambio de experiencias, } \\
\text { conocimientos,... }\end{array}$ & 2,83 & 3,24 & $\begin{array}{r}0,05 \\
1<2\end{array}$ & 4,36 & 4,25 & - \\
\hline
\end{tabular}

Tabla VIII.Valoración del dominio actual y de de la necesidad de dominio de organizar y gestionar eventos científicos según la experiencia.

\begin{tabular}{|c|c|c|c|c|c|c|}
\hline \multirow{2}{*}{$\begin{array}{l}\text { CI3: Elaborar material científico actual y } \\
\text { relevante para la docencia, para la } \\
\text { institución y para el propio avance } \\
\text { científico de su área de conocimiento }\end{array}$} & \multicolumn{3}{|c|}{$\begin{array}{c}\text { Dominio actual } \\
\text { según experiencia }\end{array}$} & \multicolumn{3}{|c|}{$\begin{array}{l}\text { Necesidad dominio } \\
\text { según experiencia }\end{array}$} \\
\hline & Novel & $\begin{array}{c}\text { Experimen } \\
\text { tado }\end{array}$ & Sig & Novel & $\begin{array}{l}\text { Experimen } \\
\text { tado }\end{array}$ & Sig \\
\hline $\begin{array}{l}\text { UC3.1.Generar producción científica de } \\
\text { documentos orientada a la publicación }\end{array}$ & 2,97 & 3,40 & - & 4,57 & 4,50 & - \\
\hline $\begin{array}{l}\text { UC3.2.Potenciar la participación y } \\
\text { colaboración de los } \\
\text { compañeros/colaboradores }\end{array}$ & 2,83 & 3,39 & $\begin{array}{l}, 008 \\
1<2\end{array}$ & 4,46 & 4,30 & - \\
\hline $\begin{array}{l}\text { UC3.3.Integrar en el trabajo propio } \\
\text { aportaciones de terceras personas }\end{array}$ & 3,19 & 3,41 & - & 4,19 & 4,20 & - \\
\hline $\begin{array}{l}\text { UC3.4.Solicitar el asesoramiento de } \\
\text { compañeros con mayor experiencia }\end{array}$ & 3,97 & 3,76 & - & 4,49 & 4,29 & - \\
\hline $\begin{array}{l}\text { UC3.5.Adaptar las ideas, producciones,... en } \\
\text { beneficio del desarrollo grupal }\end{array}$ & 3,21 & 3,38 & - & 4,35 & 4,22 & - \\
\hline
\end{tabular}

Tabla IX. Valoración del domino actual y de de la necesidad de dominio de la elaboración de material científico según la experiencia. 


\begin{tabular}{|c|c|c|c|c|c|c|}
\hline \multirow{2}{*}{$\begin{array}{l}\text { CI4: Comunicar y difundir conocimientos, } \\
\text { avances científicos, resultados de } \\
\text { proyectos de investigación e } \\
\text { innovación,... a nivel nacional e } \\
\text { internacional }\end{array}$} & \multicolumn{3}{|c|}{$\begin{array}{c}\text { Dominio actual } \\
\text { según experiencia }\end{array}$} & \multicolumn{3}{|c|}{$\begin{array}{l}\text { Necesidad dominio } \\
\text { según experiencia }\end{array}$} \\
\hline & Novel & $\begin{array}{l}\text { Experiment } \\
\text { ado }\end{array}$ & Sig & Novel & $\begin{array}{c}\text { Experimen } \\
\text { tado }\end{array}$ & Sig \\
\hline $\begin{array}{l}\text { UC4.1.Desarrollar un programa de difusión } \\
\text { múltiple de la actividad investigadora y } \\
\text { de las producciones generadas }\end{array}$ & 2,33 & 2,60 & - & 4,12 & 4,22 & - \\
\hline $\begin{array}{l}\text { UC4.2.Participar en congresos para difundir } \\
\text { el conocimiento generado }\end{array}$ & 2,97 & 3,77 & $\begin{array}{l}, 000 \\
1<2\end{array}$ & 4,15 & 4,32 & - \\
\hline $\begin{array}{l}\text { UC4.3.Publicar en editoriales, nacionales e } \\
\text { internacionales, de impacto y prestigio } \\
\text { reconocido }\end{array}$ & 2,53 & 3,03 & $\begin{array}{l}, 033 \\
1<2\end{array}$ & 4,30 & 4,38 & - \\
\hline $\begin{array}{l}\text { UC4.4.Publicar en revistas, nacionales e } \\
\text { internacionales, de impacto y prestigio } \\
\text { reconocido }\end{array}$ & 2,48 & 3,14 & $\begin{array}{l}, 008 \\
1<2\end{array}$ & 4,38 & 4,50 & - \\
\hline $\begin{array}{l}\text { UC4.5.Adecuar el discurso en función del } \\
\text { público destinatario }\end{array}$ & 3,32 & 3,83 & - & 4,41 & 4,34 & - \\
\hline
\end{tabular}

Tabla X. Valoración del dominio actual y del dominio necesario de la difusión y comunicación del conocimiento según la experiencia del profesor. 